\title{
Strates
}

STRATES Matériaux pour la recherche en sciences sociales

$12 \mid 2006$

Nouvelles tensions impériales et recompositions en Europe centrale, orientale, et CEI

\section{Les fonds structurels. Défis et enjeux du développement européen}

\section{François Bafoil}

\section{(2) OpenEdition}

\section{Journals}

Édition électronique

URL : http://journals.openedition.org/strates/2112

DOI : $10.4000 /$ strates. 2112

ISSN : $1777-5442$

Éditeur

Laboratoire Ladyss

Édition imprimée

Date de publication : 31 décembre 2006

ISSN : 0768-8067

Référence électronique

François Bafoil, «Les fonds structurels. Défis et enjeux du développement européen », Strates [En ligne], 12 | 2006, mis en ligne le 20 juillet 2007, consulté le 08 septembre 2020. URL : http:// journals.openedition.org/strates/2112 ; DOI : https://doi.org/10.4000/strates.2112

Ce document a été généré automatiquement le 8 septembre 2020.

Tous droits réservés 


\title{
Les fonds structurels. Défis et enjeux du développement européen
}

\author{
François Bafoil
}

$1 \quad$ Sur les vingt ans écoulés, les différents fonds structurels ont montré leurs capacités à relever les défis du développement dans la mesure où ils ont largement participé de la croissance des pays concernés: les quatre "pays de la cohésion» (Portugal, Grèce, Espagne, Irlande) ont vu en effet de 1992 à 1999 leur PIB moyen passer de $68 \%$ à $79 \%$ de la moyenne communautaire, (le Portugal passant de $62 \%$ à $69 \%$ et l'Espagne de $69 \%$ à $76 \%$ ). Comme l'a montré le deuxième rapport sur la cohésion en $2001^{1}$, la première conclusion à en tirer est bien de reconnaître la dynamique de convergence européenne sous l'effet des aides communautaires. Mais, et c'est la seconde conclusion tout aussi importante que la première, l'écart entre les régions les plus riches et les régions les plus pauvres à l'intérieur de chaque État s'est accru. En d'autres termes, il y a eu concentration de la dynamique de la croissance, aux dépends de certaines régions périphériques. Approfondissant ce constat, on a pu montrer que si indéniablement les instruments communautaires favorisaient l'afflux d'investissements directs étrangers dans l'Union européenne (UE), et jouaient donc l'effet de levier attendu, néanmoins les régions initialement plus riches en bénéficiaient davantage que les régions les plus pauvres. Le critère décisif semble être, en ce cas, l'appartenance aux États, les plus riches d'entre eux dotant davantage leurs régions que les plus pauvres ${ }^{2}$. Or, avec l'entrée dans l'UE en mai 2004 de dix nouveaux membres, les écarts s'accroissent et l'ordre de grandeur des aides requises est tout autre. C'est ce que révèle le troisième rapport sur la Cohésion, en date de février 2004. Si l'on inclut la Roumanie et la Bulgarie, qui devraient rejoindre l'UE avant la fin de la décennie, l'écart entre la région la plus riche et la région la plus pauvre d'Europe va passer de 5,5 (UE à 15) à plus de 10 (à 27). Cela signifie que si 73 millions de citoyens vivaient dans des régions défavorisées dans l'UE à 15, viennent maintenant s'y ajouter dans l'UE à 25, 69 millions sur les 74,5 millions de nouveaux citoyens ${ }^{3}$. Quant au PIB des moins favorisés des 15, il se situait à $68 \%$ de la moyenne européenne; avec les 25 , il se situe aux alentours de $40 \%$. Les auteurs du rapport concluaient déjà en 2001 que deux générations seraient nécessaires pour éliminer les retards dans l'UE élargie, ces affirmations recouvrant bien 
sûr d'importantes différences selon que l'on se penche sur le cas des Baltes, de l'Europe centrale ou de l'Europe orientale. C'est dire l'importance des différentiels qui séparent l'UE d'avant le 1er mai et l'actuelle. D'où la question non seulement des budgets requis ${ }^{5}$ mais d'abord de la pertinence des outils de mise en œuvre des fonds structurels dans des pays affichant des traits socio-économiques très différents de ceux qui ont forgé ces instruments.

Fonds structurels et fonds de pré-adhésionLes fonds structurels

2 Ces différents outils ont, en effet, été conçus dans un type donné d'économie, celle de l'Ouest, afin de favoriser le règlement de questions propres au développement ouesteuropéen, et à une période donnée, celle des décennies qui ont suivi la fin de la guerre. Pour cette raison, ils ont été construits au cours du temps, en fonction de l'urgence des questions à résoudre, à commencer par le premier d'entre eux, le Fonds social européen (FSE). Son objectif était dès 1957 de régler les questions de l'emploi en France et en Belgique. Au début des années 1960, la politique agricole a été organisée autour du Fonds d'orientation de la politique agricole avec une section garantie pour la Pac et une seconde, dite d'orientation, pour les fonds structurels (Feoga). Le Fonds de développement régional, le Feder, a été conçu plus tard, dans les années 1970 quand se sont posées les questions liées à la crise économique et sociale des industries lourdes. Le dernier outil, celui de la pêche (Ifop), n'a vu le jour qu'en 1993 lorsque la façade maritime de l'UE s'est considérablement augmentée grâce à l'intégration de la Suède ou de la Finlande. La question de la rationalisation de la gestion des ressources halieutiques s'est posée également en termes de reconversion des activités portuaires et de requalification des travailleurs de la mer. Conçus dans le temps pour remédier aux déséquilibres induits par le développement et pour adapter les économies et les sociétés aux défis de la modernisation en continu, ces outils ont été, de surcroît, améliorés à plusieurs reprises au cours des quatre dernières décennies. À chaque grande vague d'élargissement, les fonds ont été perfectionnés, qu'il s'agisse du Feder lors de l'intégration de la Grande-Bretagne, de l'Ifop comme on l'a dit au début de la décennie 1990 ou du développement rural lorsque se sont posées avec acuité les questions de la conditionnalité environnementale et plus généralement les options alternatives au «tout agricole». Il n'est pas jusqu'au premier Fonds, le FSE, qui n'ait connu d'importantes inflexions avec la prise en compte des obligations liées à la formation tout au long de la vie, l'égalité entre les hommes et les femmes ou encore la formation en alternance.

3 L'arrivée de dix nouveaux membres a-t-elle donné lieu à la mise au point de nouvelles stratégies? Dans un sens oui, si l'on considère les trois instruments de pré-adhésion Phare, Sapard (Special Program for Agriculture and Rural Development) et Ispa (Instrument for Structural Policies for Pre Accession). Avec eux, la Commission a cherché à disposer d'instruments facilitant le rattrapage des retards les plus criants, notamment en matière d'infrastructure. Les sommes transférées ont été conséquentes ${ }^{6}$, le nombre de jumelages signé, également. Pour cette raison, on peut penser qu'il s'est agi de l'élargissement le mieux préparé de l'UE $^{7}$ dans la mesure où à côté de ces fonds, c'est tout un système d'accompagnement (twining) et de contrôle (monitoring) qui s'est mis en place. Pourtant, au-delà du listing des initiatives entreprises et des rapports tous plus ou moins élogieux, toute la question est de bien définir ce que l'on entend par apprentissage et surtout d'évaluer son ampleur.

Les fonds de pré-adhésion 
Rappelons rapidement comment se sont présentés ces fonds de pré-adhésion. Le programme Phare initialement destiné à la Pologne et à la Hongrie a été très vite étendu aux autres candidats (Bulgarie et Roumanie compris). ${ }^{8}$. En 1997, il a été profondément modifié, notamment en raison des critiques qui ont souligné le manque de ressources humaines compétentes, le retard dans la mise en place des projets et le fait, surtout, que les projets acceptés étaient déconnectés des programmes et des budgets régionaux. Plutôt que de continuer à laisser les candidats proposer des projets financés au coup par coup et surtout de faible envergure (demand driven), la commission a pris la décision d'inscrire les aides communautaires dans le projet général de l'accession (accession driven). Dès lors, les projets ont été financés en fonction de priorités définies dans le partenariat d'accession signé avec chaque pays. En 2000, ils ont été établis conformément à l'acquis communautaire. La notion d'institution building (renforcement institutionnel) regroupant $30 \%$ des fonds a ainsi été forgée pour essayer de combler le déficit institutionnel important et l'absence de routines administratives nécessaires à la bonne gestion des fonds ${ }^{9}$. Les aides aux infrastructures ont, quant à elles, regroupé $70 \%$ des fonds Phare pour soutenir des projets d'au moins 2 millions d'euros ${ }^{10}$.

5 Dans la foulée, au début du millénaire deux autres programmes de pré-adhésion ont vu le jour, Ispa pour les infrastructures et Sapard pour le développement rural. Ces deux programmes ont connu des débuts difficiles dus principalement à la lente accréditation des agences publiques en charge de la gestion des fonds et de la mise en œuvre des projets. Un rapport établi en Pologne en avril 2003 par la Chambre supérieure de contrôle a conclu au très faible niveau de consommation des fonds par les bénéficiaires finals, soit $0,15 \%$, en raison notamment de l'insuffisance des capacités administratives, de l'inefficacité des porteurs de projets locaux et du changement constant de personnel administratif. En ce qui concerne Sapard, les critiques ont porté principalement sur la lourdeur des procédures de l'UE et sa lenteur à reconnaître les agences de mise en œuvre, de gestion et de contrôle, la bureaucratie extrême des dossiers, la lenteur de l'acceptation des dossiers. Dans un premier temps, les succès ont été enregistrés auprès des communes qui ont consommé les fonds pour des travaux d'infrastructure rurale (adduction d'eau, viabilité des champs, etc.). Plus tard, les exploitants ont su prendre à leur compte ce programme pour améliorer les exploitations. Cependant, un moindre succès a été enregistré avec les filières de transformation qui n'ont pas su bénéficier des fonds. De surcroit, on doit noter qu'avec ISPA, il s'agit d'un programme centralisé, peu à même de favoriser l'apprentissage régional et que dans les deux programmes de pré-adhésion, la gestion budgétaire est annuelle, à la différence des fonds structurels, qui sont pluriannuels. Ils réclament donc une tout autre structure de gestion et de contrôle. Enfin, la notion de stratégie différencie largement les deux sources de financements (soit les programmes de pré-adhésion et les fonds structurels), dans la mesure où les fonds structurels, pour être délivrés, doivent s'inscrire dans la politique sectorielle ministérielle ou régionale et s'étendre sur le long terme. La question est bien celle de la capacité des acteurs publics à articuler une stratégie en isolant les points forts et les points faibles que les fonds structurels pourraient à la fois renforcer et réduire sur le long terme, en partenariat avec les autres acteurs non publics, privés et associatifs. La question stratégique est donc étroitement liée à cet autre aspect décisif, qu'est la capacité d'absorption. 
6 On doit noter par ailleurs que le troisième rapport sur la Cohésion paru en janvier 2004 propose pour la prochaine programmation (celle de 2006-2013) une simplification des fonds. De quatre (FSE, Feder, Ifop et Feoga O), ils passent à deux puisque ne seront conservés que le FSE et le Feder, tandis que le fond pour le développement rural est confondu avec le second pilier de la PAC ainsi que l'Ifop ${ }^{11}$. Cela posé, les nouvelles fonctions de ces fonds ne sont guère différentes des précédentes, si l'on excepte l'accent mis sur la notion de convergence économique, sociale et territoriale. En tous les cas, les particularités avancées par les situations est-européennes ne font pas l'objet d'une réflexion, comme si la notion de rattrapage (catch up) avait réglé le problème une bonne fois pour toutes. Ces sociétés sont en retard de développement, c'est tout; et de la même manière qu'en 1990, de nombreux observateurs pensaient qu'ils suffisaient de dresser l'inventaire des servitudes de l'économie de type soviétique pour brandir la pièce manquante censée remettre la "machine » en marche, de même quinze ans plus tard, on s'appuie sur des indices reflétant le retard par rapport aux moyennes de l'UE à 15 pour mieux vanter les mérites des outils ou plutôt ne pas poser la question de leur validité. Il n'est question finalement que d'adaptation et de reprise, jamais d'innovation en face de situations uniques en Europe. L'apprentissage n'est guère évalué. La représentation dominante est toujours aussi mécanique qu'auparavant et la mesure des efforts, strictement réduite à l'examen des indices quantitatifs. La procédure d'examen (screening) et de suivi (monitoring) de toutes les étapes de l'adoption de l'Acquis communautaire (plus de 80000 pages pour chaque pays) a été rigoureusement appliquée et produit nombre de rapports. Quant aux travaux empiriques sur les questions soulevées par le transfert, l'adaptation, l'apprentissage, ils ont été complètement manquants.

7 Si l'on considère maintenant quelques situations des nouveaux membres, comment évaluer la pertinence des fonds ${ }^{12}$ ? Limitons nous aux seuls outils Feder et FSE (la commission ayant proposé dans son rapport de février 2004 de ne conserver que ces deux outils, et de fondre le Feoga-O ainsi que l'Ifop au sein d'une vaste politique agricole et rurale) ${ }^{13}$.

Examen de la préparation aux fonds structurels

8 À propos du Feder, trois questions se posent qui ont trait à la vocation même du Feder, comme outil du développement. Le Feder, vise le renforcement du tissu des PME, celui des milieux de la recherche et de l'innovation et enfin celui des infrastructures. Soulignons quelques difficultés propres au plus important programme, celui de la compétitivité.

9 Les PME, qui ont surgi en très grand nombre après 1989, ont assuré un dynamisme sans précédent aux économies des pays est-européens. Pourtant, elles présentent des traits largement différents de ceux de leurs homologues d'Europe de l'Ouest. L'intense mouvement de création et de destruction qui affecte le secteur des PME depuis 1990 témoigne de l'instabilité chronique des marchés. Le nombre moyen d'employés est bien moindre. En Pologne, on compte en moyenne trois salariés lorsqu'il est de neuf à l'Ouest. Les qualifications y sont, de surcroît, très basses.

10 Cela rend illusoires les paris faits sur l'informatisation à court terme des tissus économiques. Enfin, les produits ne sont pas de forte valeur ajoutée. Cela, également, rend peu crédibles les projets de transferts des hautes technologies. Les PME sont davantage actives dans les secteurs de la réparation, du service de proximité, du commerce de détail. L'aide publique ne peut donc pas porter sur elles, sinon 
marginalement. En revanche, l'effort de soutien aux PME passe par le choix politique d'isoler les entreprises qui sont susceptibles d'assurer la croissance, autrement dit celles qui exportent déjà. Le risque, bien évidemment, est d'approfondir les fractures qui se font jour entre des pôles développés ou en forte croissance et d'autres, qu'aucun facteur de redéveloppement ne favorise. En Pologne, sur les trois millions de PME recensées en 2004, à peine deux millions sont dites en activité et sur ce chiffre de deux millions, seulement 14000 d'entre elles sont actives à l'exportation. Ce sont ces dernières et elles seules qui peuvent prétendre être éligibles aux programmes de soutien européen de grande envergure, car ce sont les seules à faire état de capacités technologiques ou d'infrastructures commerciales de poids. On comprend dès lors les effets politiques possibles d'une telle décision si d'aventure les aides européennes ne devaient être accordées qu'à elles, et l'on peut s'interroger sur l'efficacité économique attendue d'un tel choix.

11 À propos du FSE, deux difficultés peuvent être mentionnées. La première difficulté concerne la stratégie pour l'emploi énoncée par la Commission et acceptée par les États membres. Elle stipule, entre autres points, la notion d'égalité des chances entre les hommes et les femmes. Or, c'est un thème méconnu des États nouveaux membres (même si l'emploi féminin était une réalité avant 1989). L'inégalité entre hommes et femmes s'est trouvée fortement accentuée après 1990 en raison du fait que les femmes ont été massivement les victimes des licenciements tout en étant écartées des mesures d'aides à la reconversion qui ont systématiquement privilégié les emplois masculins. Enfin, à compter de 1990, les prestations sociales ont diminué, réduisant encore davantage les marges d'autonomie des femmes.

Le second axe de la stratégie européenne qui n'a guère été intégré, à tout le moins avec grande difficulté, vise la formation, qu'elle soit initiale ou continue. La question de l'abandon scolaire n'a que rarement été prise en compte, de même que l'illettrisme dont les régimes précédents s'étaient félicités de l'éradication. Les statistiques sont manquantes sur ce point. L'aspect de la formation en alternance a été écarté pour beaucoup de nouveaux candidats, au motif que les entreprises susceptibles de permettre cette alternance ont massivement disparu. Quant à la formation tout au long de la vie, il s'agit là d'un concept entièrement nouveau, difficilement intégrable dans les stratégies nationales ou régionales. Les dispositifs et financements nationaux ne concernent souvent que les chômeurs. Quant au marché privé de la formation, il est embryonnaire et intégralement privé : d'où la question non seulement du financement, mais de l'accès aux centres de formation sur tout le territoire.

Les défis de l'adaptation : un enjeu politique

13 Ainsi, les défis sont immenses. Si l'on essaie d'y répondre en termes d'objectifs à atteindre, il s'agit de créer des unités Feder et FSE dans tous les ministères de l'économie et du travail et chez les grands bénéficiaires que sont les services de l'emploi et les ministères de l'éducation; donc de former des agents; de définir des procédures administratives financières de contrôle; d'adapter la législation (notamment sur les marchés publics et le conventionnement); de mettre au point des manuels de procédure et des outils de suivi (sous la forme d'outils informatique et de monitoring), enfin de former des techniciens de l'évaluation.

14 À cela vient s'ajouter la nécessité de construire les partenariats tant au niveau des ministères de l'emploi qu'entre les ministères concernés (éducation, finances, développement régional, etc.) et au niveau des partenaires privés et des associations. La 
maîtrise de l'exercice complexe des procédures de l'UE impliquant la construction des programmes sur la base de l'énoncé des priorités et de mesures de l'intervention, l'affectation et la répartition des budgets, les négociations interministérielles, cette maîtrise a souvent bloqué toute autre réflexion portant sur l'organisation administrative, la question des compétences, la définition des objectifs à long terme, le contrôle.

15 Autre axe dont le déficit est criant: le dialogue social. À cela plusieurs raisons, à commencer par le profond discrédit dans lequel sont tombés les syndicats d'ancien régime inféodés aux partis communistes en place et jamais préoccupés avant 1989 de la défense des intérêts des travailleurs. Quinze ans après la chute du communisme, le paysage des syndicats est contrasté par pays, mais d'une manière générale, on peut affirmer qu'ils ne se trouvent que dans les secteurs publics, eux-mêmes en régression. Les conflits ne concernent que quelques catégories de salariés: les paysans, et les salariés des secteurs publics de santé ou encore d'éducation. Quant aux syndicats d'employeurs, ils se distinguent par leur faiblesse, voire leur inexistence. Pourtant, il semble que la nécessité d'un dialogue social ne soit pas à l'ordre du jour, ce que reflète le très faible niveau de conventionnement, que ce soit au niveau sectoriel ou au niveau local. En face de cette désorganisation des acteurs sociaux, les États n'ont pas cherché à intégrer les partenaires au jeu politique. Ils les ont confinés au début des années 1990 à la discussion autour des minima sociaux dans des forums intitulés pompeusement "forums tripartites" et les ont écartés purement et simplement du montage des programmes au titre des fonds structurels.

16 En somme, l'enjeu est largement politique car il concerne directement la question de la modernisation de l'État. Cette modernisation est en cours. La dynamique est déjà très importante sous l'effet à la fois de l'adoption intégrale de l'acquis communautaire et de l'adaptation aux exigences de la gestion des fonds européens. Pourtant en de nombreux endroits, les équipes politiques à la tête des administrations se distinguent par la permanence de réflexes inappropriés comme en témoigne la pratique à grande échelle du spoil system. Toutes les administrations souffrent en effet de ce phénomène, qui consiste à renvoyer à chaque élection les individus les plus gradés, et souvent également les plus formés, notamment sur fonds européens, sous prétexte que tous ont bénéficié, en principe, des avantages de la précédente équipe.

17 La difficulté et la lenteur des autorités publiques et européennes à promouvoir un statut de la fonction publique et du fonctionnaire ont pu être expliqués, par ailleurs, par le fait que l'UE n'avait en rien assisté les nouveaux membres sur ce point. Or, si le critère de bonne gouvernance administrative figure dans l'Agenda 2000 au même titre que la démocratie et le marché, jamais l'UE n'a fourni autre chose que de vagues prescriptions faisant référence à la prévisibilité, la responsabilité, la transparence et l'efficacité. Du coup, faute de disposer d'un cadre précis de la "bonne administration ", les autorités politiques locales ont eu tout intérêt à ne pas modifier leur propre édifice. Grâce au maintien de la loyauté comme ressort de l'action administrative, elles peuvent tenir en respect les oppositions et s'assurer de solides fiefs politiques ${ }^{14}$.

De surcroît, les administrations centrales rechignent à partager leurs prérogatives avec les régions en matière de politiques sectorielles, ne serait-ce que pour conserver pour leur profit la plus grande part des fonds européens. Quant aux administrations régionales, elles ont le même comportement à l'encontre de leurs partenaires locaux, en ne les associant que trop rarement à la définition de la stratégie régionale. Toutes 
hésitent à lancer des coopérations avec les acteurs privés, notamment associatifs et aucune administration centrale ou régionale ne paie correctement ses employés. Or, en cette intense période de gestion des fonds qui correspond également à un important chômage des jeunes, de plus en plus de qualifications sont requises pour ces derniers, et notamment ceux en charge des dossiers européens, en échange de salaires dérisoires.

19 Face à cette incurie des administrations traditionnelles, on peut se demander si la solution ne passerait pas par la mise sur pied d'agences de gestion des fonds européens, de statut semi-public, dont les règles statutaires restent à inventer. Semblables agences existent dans les pays anglo-saxons ainsi qu'en Grèce et au Portugal ${ }^{15}$. Plusieurs donnent des résultats positifs si l'on s'en tient, du moins, au succès des fonds structurels en ces pays ${ }^{16}$. La question est néanmoins de savoir si tous les citoyens pourront être traités sur un pied d'égalité par ces agences. On sait dans le cas rural par exemple, qu'elles ne pourront être au service que d'une partie des exploitants, ceux disposant de plus de 20 hectares, et prêts à s'acquitter de coûts d'entrée élevés. Autrement dit, de telles agences ne s'adresseront pas à la grande partie des paysans dont plus de $50 \%$ en Pologne ont moins de 5 hectares. Le soutien à la compétitivité pourrait s'accompagner dans ce cas d'une très forte dégradation des conditions de vie de la majorité.

Conclusions provisoires

Trois questions peuvent être soulevées en conclusion de cette esquisse rapide de l'état de préparation des fonds.

21 D'abord sur les enseignements à tirer des expériences en cours. Une réflexion intense doit désormais voir le jour à propos de la pertinence de l'application des outils de l'UE à des économies largement divergentes de ce que l'UE a eu jusqu'ici à intégrer. Les enseignements tirés des expériences des nouveaux membres seront très importants pour les candidats, notamment pour tout ce qui concerne l'agriculture et le développement rural. Mais sans doute conviendrait-il de ne pas se limiter à l'analyse d'approches quantitatives de l'apprentissage et de réfléchir à la mise au point d'indicateurs plus complexes impliquant la réflexion sur les modes de vie, la justesse et donc la légitimité des interventions. La question de la légitimité des interventions de l'UE est capitale pour tenter de dépasser le clivage qui semble s'approfondir entre les populations et les élites européennes.

Centraliser ou décentraliser? À l'issue de son expérience en Pologne comme responsable de l'évaluation ex ante et comme expert en 2002-2003, l'auteur de cet article en vient à se demander si l'on n'a pas trop demandé d'efforts aux candidats compte tenu de l'absence de ressources administratives. N'aurait-il pas mieux valu être conséquent jusqu'au bout, centraliser la programmation, comme l'on a centralisé la procédure de la construction du programme régional, et finalement, imposer d'en haut des programmes prioritairement orientés vers les infrastructures de transports? La période est trop courte et les exigences trop élevées. Il est à craindre que ne dominent un grand nombre de petits projets et pourtant les infrastructures sont encore largement manquantes.

23 Certes, on pourra toujours craindre que la démocratie et l'apprentissage ne fassent les frais d'une telle centralisation. Ce serait oublier le lent et décisif progrès de «l'européanisation » au travers de la préparation qui vient d'avoir lieu et que la mise en place de grands programmes d'intervention ne pourra que renforcer. L'important est surtout que des interventions publiques aient lieu pour permettre aux citoyens de 
se rendre compte de visu que tous les efforts qui ont été consentis depuis près de 15 ans pour intégrer l'UE ne l'ont pas été en vain. Les fonds doivent être consommés par souci de légitimation de l'action publique, mais aussi parce que ne pas consommer signifierait pour certains, trop nombreux en Europe de l'Ouest, que l'argent est versé à perte pour les nouveaux membres et, qu'en conséquence, il convient de redéfinir la priorité des attributions.

En revanche, une réflexion très importante devrait maintenant avoir lieu sur les agences de mise en œuvre. Réflexion qu'il revient à la Commission et aux membres de conduire pour énoncer les règles statutaires de bonne gestion d'agences de délégation semi-publiques en charge des fonds structurels. Les avantages de telles agences seraient, outre d'assurer la gestion correcte des fonds, d'offrir une rémunération élevée pour les salariés, que les administrations publiques sont dans l'incapacité de garantir aux jeunes diplômés qu'elles entendent employer, et d'être le creuset de la démocratie de demain.

\section{NOTES}

1. Référence : www.europa.eu.int/inforregio

2. J. Fayolle, A. Lecuyer, «Croissance régionale, appartenance nationale et fonds structurels européens : un bilan d'étape ", Revue de l'OFCE, n 73, avril 2000.

3. Rapport, février 2004, cf. www.europa.eu.int/inforegio (consulté en 04/06). Une région est dite défavorisée lorsque son PIB régional est inférieur à $75 \%$ de la moyenne communautaire et se trouve ainsi éligible aux fonds structurels au titre de l'objectif 1 . Ces régions souffrent d'un chômage élevé, de faibles infrastructures et de faibles investissements, enfin, d'un manque de services. Le $2^{\mathrm{e}}$ objectif concerne les régions faisant face à des difficultés de redéploiement industriel ou rural. L'objectif 3 sous-tend les politiques de développement de l'éducation, de la formation, et de l'emploi. En montants financiers, les fonds structurels représentent pour la programmation de 2000-2006 environ 213 milliards d'euros, soit environ $36 \%$ du budget total de l'UE. Dans la prochaine programmation qui s'étendra de 2007 à 2013, les fonds devraient être réduits à deux (FEDER et FSE) et le zonage, disparaître.

4. Le PIB de la République tchèque est environ à $60 \%$ de la moyenne communautaire, mais celui de la Pologne, de la Lituanie et de l'Estonie, à $40 \%$.

5. Cf. J. Le Cacheux « Negotiating the Medium Term financial Perspectives for the enlarged EU: the future of the european budget », Revue de l'OFCE, special issue, «The New European Union Enlargement », avril 2004, p. 175-189.

6. Pour la période 2000-2006, les montants financiers alloués ont été pour Phare de 1560 millions d'euros, pour Ispa de 1040 millions et pour Sapard de 520 millions. En comparaison, les fonds structurels et de cohésion sont de 40 milliards d'euros.

7. J. d'Haussonville, «Les processus d'adhésion : cet élargissement est-il le mieux préparé de l'histoire de l'UE ? ", Pouvoir, 2003, p. 5-39. 
8. M. Goinard, « Le programme communautaire Phare : d'une assistance classique à un système d'apprentissage des mécanismes de l'UE ", Allemagne d'Aujourd'hui, n 166 , octdéc. 2003, p. 39-53.

9. Pour faciliter les transferts d'Ouest en Est, des jumelages ont été établis entre régions (les Twining) consistant notamment en la mise à disposition de fonctionnaires d'un État membre durant une période allant jusqu'à 18 mois (Conseiller pré-adhésion, entouré d'experts moyen terme et long terme).

10. Les principaux programmes ont concerné les infrastructures, les aides au secteur privé, l'agriculture, la formation et l'éducation, la protection de l'environnement et la protection sociale.

11. www.europa.eu.int/inforegio (consulté en 04/06).

12. Cf. F. Bafoil, E. Lhomel, « La préparation aux fonds structurels de l'UE, Les exemples de la Pologne ", Le Courrier des Pays de l'Est, mars 2003, p. 28-38, 2003, cf. le numéro spécial de la Revue d'études comparatives Est/Ouest, 2003.

13. «Les jumelages institutionnels au titre du programme Phare. Limites d'une intervention et apprentissage collectifs ", F Bafoil, R. Guyet, F. Beaumelou, G. Lepesant, E. Lhomel, C. Perron, numéro spécial consacré aux fonds structurels, Critique Internationale, 2004, n 25 ; F. Bafoil, « Les instruments de l'élargissement de l'Union européenne » p. 117-183. On trouvera dans ce numéro spécial, outre cet article collectif reprenant l'essentiel des réflexions du groupe qui s'est tenu en 2004 au Ceri sur les fonds structurels, des contributions portant sur le développement régional, sur les politiques de l'emploi, sur les politiques de transport.

14. A. Dimitrova, «Enlargement, Institution Building and the EU's administrative Capacity Requirement », West European Politics, vol. 25, oct. 2002, 4, p. 171-190.

15. Cf. F. Bafoil \& B. Hibou, « Européanisation : quelles mutations des administrations publiques et des modes de gouvernement? Une comparaison Europe du Sud, Europe de l'Est » Études du CERI, décembre 2003.

16. M. Flinders, , " Distributed public governance in the european Union », Journal of European Public Policy, june 2004, 11, 3, p. 520-544.

\section{RÉSUMÉS}

Les fonds structurels ont été conçus dans l'Union européenne pour garantir le processus de décollage des nouveaux membres. Ils ont été mis en place dans quelques régions reculées présentant des conditions socio-économiques profondément différentes de celles pour lesquelles ces outils européens avaient été initialement mis en place. Cependant, la mise en place des fonds structurels suppose un large dialogue social entre les différents acteurs et en Europe de l'Est les acteurs principaux du dialogue social manquent. Cet article précise quelques difficultés concernant les anomalies socio-économiques entre les deux parties de l'Europe en insistant sur l'héritage historique.

Françoise Bafoil. Structurals funds: defis and Stakes in European development

Structural funds were designed in the European Union to guarantee the catch up process of the new members. They have to be implemented in some backward regions presenting some socio 
economic features deeply different from those to which these European tool were initially designed. Moreover, the implementation of the structural funds claims for a large social dialogue between the different actors, and in Eastern Europe the main actors of the social dialogue are missing. This article points out some difficulties about the socio economic discrepancies between the two parts of Europe insisting on the historical legacies.

\section{AUTEUR}

FRANÇOIS BAFOIL

Chercheur, Ceri, CNRS, bafoil@ceri-sciences-po.org 